\title{
ON THE LOCAL DENSITY PROBLEM FOR GRAPHS OF GIVEN ODD-GIRTH
}

\author{
WIEBKE BEDENKNECHT, GUILHERME OLIVEIRA MOTA, CHRISTIAN REIHER, \\ AND MATHIAS SCHACHT
}

\begin{abstract}
Erdős conjectured that every $n$-vertex triangle-free graph contains a subset of $\lfloor n / 2\rfloor$ vertices that spans at most $n^{2} / 50$ edges. Extending a recent result of Norin and Yepremyan, we confirm this conjecture for graphs homomorphic to so-called Andrásfai graphs. As a consequence, Erdős' conjecture holds for every triangle-free graph $G$ with minimum degree $\delta(G)>10 n / 29$ and if $\chi(G) \leqslant 3$ the degree condition can be relaxed to $\delta(G)>n / 3$. In fact, we obtain a more general result for graphs of higher odd-girth.
\end{abstract}

\section{§1. INTRODUCTION}

We say an $n$-vertex graph $G$ is $(\alpha, \beta)$-dense if every subset of $\lfloor\alpha n\rfloor$ vertices spans more than $\beta n^{2}$ edges. Given $\alpha \in(0,1]$, Erdős, Faudree, Rousseau, and Schelp [8] asked for the minimum $\beta=\beta(\alpha)$ such that every $(\alpha, \beta)$-dense graph contains a triangle. For example, Mantel's theorem asserts that $\beta(1)=1 / 4$. More generally, Erdös et al. conjectured that for $\alpha \geqslant 17 / 30$ the balanced complete bipartite graph gives the best lower bound for the function $\beta(\alpha)$, which leads to

$$
\beta(\alpha)=\frac{1}{4}(2 \alpha-1) .
$$

The same authors verified this conjecture for $\alpha \geqslant 0.648$ and the best result in this direction is due to Krivelevich [12], who verified it for every $\alpha \geqslant 3 / 5$.

We say a graph $G$ is a blow-up of some graph $F$, if there exists a graph homomorphism $\varphi: G \rightarrow F$ with the property $\{x, y\} \in E(G)$ if and only if $\{\varphi(x), \varphi(y)\} \in E(F)$. Moreover, a blow-up is balanced if the preimages $\varphi^{-1}(v)$ of all vertices $v \in V(F)$ have the same size. For $\alpha<17 / 30$ balanced blow-ups of the 5-cycle yield a better lower bound for $\beta(\alpha)$ and Erdős et al. conjectured

$$
\beta(\alpha)=\frac{1}{25}(5 \alpha-2)
$$

for $\alpha \in[53 / 120,17 / 30]$. For $\alpha<53 / 120$ it is known that balanced blow-ups of the Andrásfai graph $F_{3}$ (see Figure 1) lead to a better bound. The special case $\beta(1 / 2)=1 / 50$ was considered before by Erdős [6] (see also [7] for a monetary bounty for this problem).

The second author was supported by FAPESP (Proc. 2013/11431-2, 2013/20733-2) and partially by CNPq (Proc. 459335/2014-6). The collaboration of the authors was supported by CAPES/DAAD PROBRAL (Proc. 430/15) and by FAPESP (Proc. 2013/03447-6). 
Conjecture 1.1 (Erdős). Every (1/2,1/50)-dense graph contains a triangle.

Currently, the best known upper bound on $\beta(1 / 2)$ is $1 / 36$ and was obtained by Krivelevich [12]. Besides the balanced blow-up of the 5-cycle, Simonovits (see, e.g., [7]) noted that balanced blow-ups of the Petersen graph yield the same lower bound for Conjecture 1.1 and, more generally, for (1.2) in the corresponding range.

Conjecture 1.1 asserts that every triangle-free $n$-vertex graph $G$ contains a subset of $\lfloor n / 2\rfloor$ vertices that spans at most $n^{2} / 50$ edges. Our first result (see Theorem 1.2 below) verifies this for graphs $G$ that are homomorpic to a triangle-free graph from a special class.

1.1. Andrásfai graphs. A well studied family of triangle-free graphs, which appear in the lower bound constructions for the function $\beta(\alpha)$ above, are the so-called Andrásfai graphs. Those graphs already appeared in the work of Erdős [5] and Andrásfai [1,2]. Andrásfai graphs are contained in several interesting graph classes. For example, they are special circulant graphs, they appear as finite subgraphs of 2-dimensional Borsuk graphs, and can be described as the complement of an appropriate power of a cycle. For our purposes it is convenient to define them as follows.

For an integer $d \geqslant 1$ the Andrásfai graph $F_{d}$ is the $d$-regular graph with vertex set

$$
V\left(F_{d}\right)=\left\{v_{1}, \ldots, v_{3 d-1}\right\},
$$

where $\left\{v_{i}, v_{j}\right\}$ forms an edge if

$$
d \leqslant|i-j| \leqslant 2 d-1
$$

Note that $F_{1}=K_{2}$ and $F_{2}=C_{5}$ (see Figure 1). It is easy to check that Andrásfai graphs are triangle-free and balanced blow-ups of these graphs play a prominent rôle in connection with extremal problems for triangle-free graphs (see, e.g., $[1-5,9,10,15]$ ).
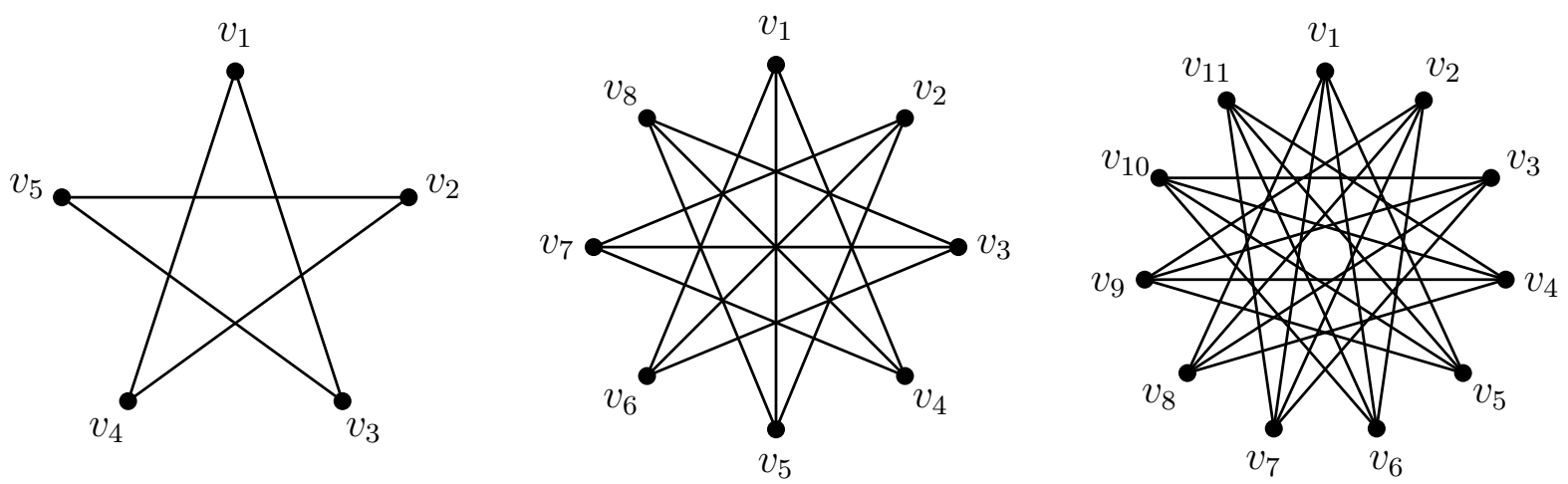

Figure 1. Andrásfai graphs $F_{2}, F_{3}$, and $F_{4}$. 
Our first result validates Conjecture 1.1 (stated in the contrapositive) for graphs homomorphic to some Andrásfai graph.

Theorem 1.2. If a graph $G$ is homomorphic to an Andrásfai graph $F_{d}$ for some integer $d \geqslant 1$, then $G$ is not $(1 / 2,1 / 50)$-dense.

Since $F_{d}$ is homomorphic to $F_{d^{\prime}}$ if and only if $d^{\prime} \geqslant d$, Theorem 1.2 extends recent work of Norin and Yepremyan [14], who obtained such a result for $n$-vertex graphs $G$ homomorphic to $F_{5}$ with the additional minimum degree assumption $\delta(G) \geqslant 5 n / 14$.

Owing to the work of Chen, Jin, and Koh [4], which asserts that every triangle-free 3-chromatic $n$-vertex graph $G$ with minimum degree $\delta(G)>n / 3$ is homomorphic to some Andrásfai graph, we deduce from Theorem 1.2 that Conjecture 1.1 holds for all such graphs $G$. Similarly, combining Theorem 1.2 with a result of Jin [10], which asserts that triangle-free graphs $G$ with $\delta(G)>10 n / 29$ are homomorphic to $F_{9}$, implies Conjecture 1.1 for those graphs as well. We summarise these direct consequences of Theorem 1.2 in the following corollary.

Corollary 1.3. Let $G$ be a triangle-free graph on $n$ vertices.

(a) If $\delta(G)>10 n / 29$, then $G$ is not $(1 / 2,1 / 50)$-dense.

(b) If $\delta(G)>n / 3$ and $\chi(G) \leqslant 3$, then $G$ is not $(1 / 2,1 / 50)$-dense.

We remark that part $(a)$ slightly improves earlier results of Krivelevich [12] and of Norin and Yepremyan [14] (see also [11] where an average degree condition was considered).

1.2. Generalised Andrásfai graphs of higher odd-girth. We consider the following straightforward variation of Andrásfai graphs of odd-girth at least $2 k+1$, i.e., graphs without odd cycles of length at most $2 k-1$. For integers $k \geqslant 2$ and $d \geqslant 1$ let $F_{d}^{k}$ be the $d$-regular graph with vertex set

$$
V\left(F_{d}^{k}\right)=\left\{v_{1}, \ldots, v_{(2 k-1)(d-1)+2}\right\},
$$

where $\left\{v_{i}, v_{j}\right\}$ forms an edge if

$$
(k-1)(d-1)+1 \leqslant|i-j| \leqslant k(d-1)+1 .
$$

In particular, for $k=2$ we recover the definition of the Andrásfai graphs from (1.3) and for general $k \geqslant 2$ we have $F_{1}^{k}=K_{2}, F_{2}^{k}=C_{2 k+1}$ and for every $d \geqslant 2$ the graph $F_{d}^{k}$ has odd-girth $2 k+1$ (see Figure 2).

Our main result generalises Theorem 1.2 for graphs of odd-girth at least $2 k+1$. In fact, the constant $\frac{1}{2(2 k+1)^{2}}$ appearing in Theorem 1.4 is best possible as balanced blow-ups of $C_{2 k+1}$ show. One can attain this bound by taking $k$ mutually independent parts of the 

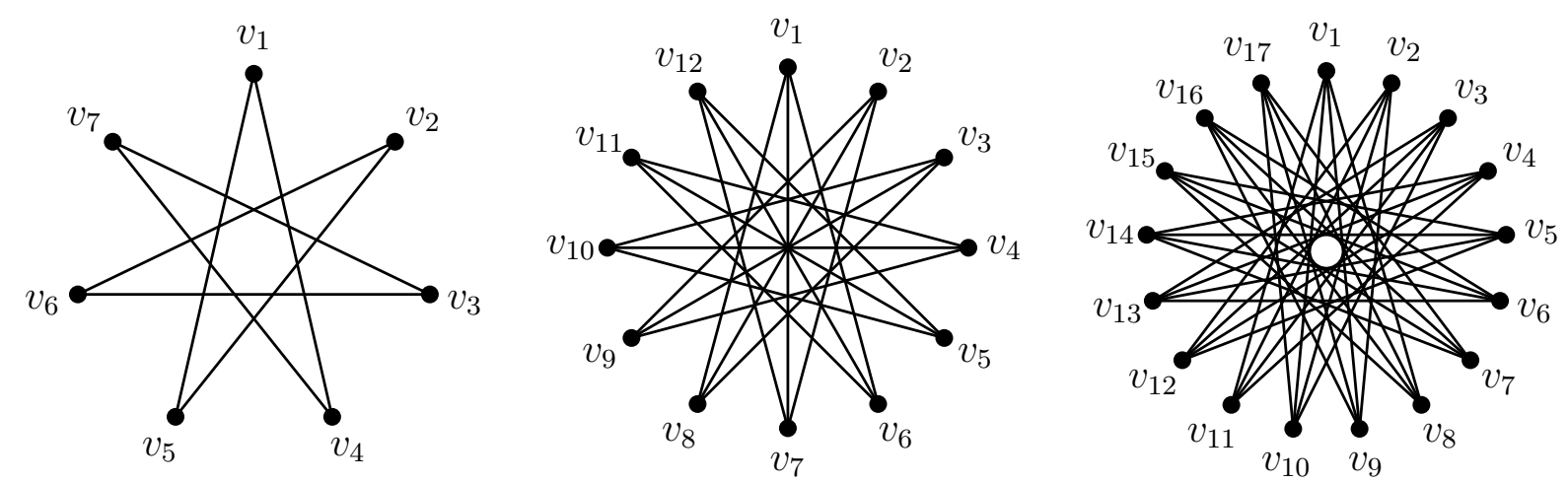

Figure 2. Generalised Andrásfai graphs $F_{2}^{3}, F_{3}^{3}$, and $F_{4}^{3}$ of odd-girth 7 .

blow-up completely and the missing $n / 2-k n /(2 k+1)$ vertices from one part, which has only edges to one of the parts that we already chose.

Theorem 1.4. If a graph $G$ is homomorphic to a generalised Andrásfai graph $F_{d}^{k}$ for some integers $k \geqslant 2$ and $d \geqslant 1$, then $G$ is not $\left(\frac{1}{2}, \frac{1}{2(2 k+1)^{2}}\right)$-dense.

Analogous to the relation between Conjecture 1.1 and Theorem 1.2 one may wonder if every $\left(\frac{1}{2}, \frac{1}{2(2 k+1)^{2}}\right)$-dense graph contains an odd cycle of length at most $2 k-1$. Letzter and Snyder [13] showed that a graph $G$ on $n$ vertices with $\delta(G)>\frac{n}{5}$ and odd-girth at least 7 is homomorphic to $F_{k}^{3}$, for some $k$. Therefore combining this result with Theorem 1.4 we get the following.

Corollary 1.5. Let $G$ be a graph with odd-girth at least 7 on $n$ vertices. If $\delta(G)>\frac{n}{5}$, then $G$ is not $\left(\frac{1}{2}, \frac{1}{98}\right)$-dense.

A similar question for even holes is not interesting, because every dense graph contains a 4-cycle.

For $k=2$ Theorem 1.4 reduces to Theorem 1.2 and the rest of this work concerns the proof of Theorem 1.4. The proof is given in Section 3 and makes use of a geometric representation of graphs homomorphic to generalised Andrásfai graphs, which we introduce in Section 2.

\section{§2. A geometric characterisation of Generalised Andrásfai graphs}

We consider graphs $G$ that are homomorphic to some generalised Andrásfai graph $F_{d}^{k}$. For the proof of Theorem 1.4 it will be convenient to work with a geometric representation of such graphs $G$. In fact, already the original definitions of Andráfai graphs in [1,5] were geometric. In that representation we will arrange the vertices of $G$ on the unit circle $\mathbb{R} / \mathbb{Z}$ and edges between two vertices $x$ and $y$ may only appear depending on their angle with 
respect to the centre of the circle (see Lemma 2.1). For the proof of Theorem 1.4 it suffices to consider edge maximal graphs $G$ that are homomorphic to $F_{d}^{k}$ for some integers $k \geqslant 2$ and $d \geqslant 1$. In other words, we may assume $G$ is a blow-up of $F_{d}^{k}$.
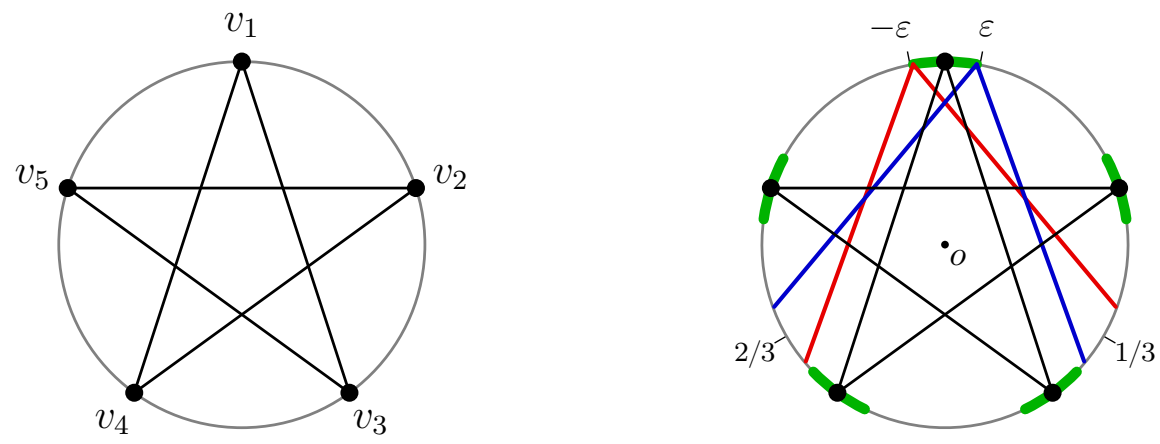

Figure 3. A copy of $F_{2}=C_{5}$ and a representation of a blow-up on the unit circle.

For example, let $G$ be a blow-up of $F_{2}=C_{5}$. One can distribute the vertices of $F_{2}$ equally spaced on the unit circle (see Figure 3). Then we place all vertices of $G$ that correspond to the blow-up class of $v_{i}$ into a small $\varepsilon$-ball around $v_{i}$ on the unit circle (cf. green arcs in Figure 3). For a sufficiently small $\varepsilon$, all vertices in an $\varepsilon$-ball around $v_{i}$ have the same neighbours and they can be characterised by having their smaller angle with respect to the centre bigger than $120^{\circ}$ (cf. red and blue lines in Figure 3). The following lemma states this fact for blow-ups of generalised Andrásfai graphs.

Lemma 2.1. If $G$ is a blow-up of a generalised Andrásfai graph $F_{d}^{k}$ for some integers $k \geqslant 2$ and $d \geqslant 1$, then the vertices of $G$ can be arranged on the unit circle $\mathbb{R} / \mathbb{Z}$ with centre o such that

$$
\{x, y\} \in E(G) \quad \Longleftrightarrow \quad \Varangle x \text { oy }>\frac{k-1}{2 k-1} \cdot 360^{\circ},
$$

where $\Varangle x o y$ denotes the smaller angle between $x$ and $y$ with respect to $o$.

We remark that conversely every graph $G=(V, E)$ with $V \subseteq \mathbb{R} / \mathbb{Z}$ satisfying (2.1) is a blow-up of $F_{d}^{k}$ for some appropriate $d \geqslant 1$. However, since this direction is not utilised here, we omit the formal proof of this observation.

Proof of Lemma 2.1. For integers $k \geqslant 2$ and $d \geqslant 1$ let $G$ be a blow-up of the generalised Andrásfai graph $F_{d}^{k}$ (defined in (1.4)) signified by some graph homomorphism $\varphi: G \rightarrow F_{d}^{k}$ and let $m=(2 k-1)(d-1)+2$ be the number of vertices of $F_{d}^{k}$. Set

$$
\varepsilon=\frac{1}{2(2 k-1) m} .
$$


For $i \in[m]$ we arrange the vertices of $G$ that are contained in $\varphi^{-1}\left(v_{i}\right)$ in the $\varepsilon$-ball around the point $\frac{i-1}{m}$. Owing to the symmetry it suffices to check that (2.1) holds for an arbitrary vertex $x \in \varphi^{-1}\left(v_{1}\right) \subseteq V(G)$.

By definition of $F_{d}^{k}$ the neighbourhood of $v_{1}$ is

$$
N\left(v_{1}\right)=\left\{v_{(k-1)(d-1)+2}, \ldots, v_{k(d-1)+2}\right\} .
$$

Note that the choice of $\varepsilon$ gives

$$
\left(\frac{(k-1)(d-1)+1}{m}-\varepsilon, \frac{k(d-1)+1}{m}+\varepsilon\right)=\left(\frac{k-1}{2 k-1}+\varepsilon, \frac{k}{2 k-1}-\varepsilon\right)
$$

and, consequently, all neighbours $y$ of $x$ are placed in the interval $\left(\frac{k-1}{2 k-1}+\varepsilon, \frac{k}{2 k-1}-\varepsilon\right)$. Since $x \in \varphi^{-1}\left(v_{1}\right)$ itself is placed in $(-\varepsilon, \varepsilon)$, this implies the forward direction of $(2.1)$. The converse direction follows from the observation

$$
\left(\frac{i-1}{m}-\varepsilon, \frac{i-1}{m}+\varepsilon\right) \cap\left(\frac{k-1}{2 k-1}-\varepsilon, \frac{k}{2 k-1}+\varepsilon\right)=\varnothing
$$

for every $i \in[m] \backslash\{(k-1)(d-1)+2, \ldots, k(d-1)+2\}$.

We close this section with a few useful estimates on the number of vertices contained in intervals of $\mathbb{R} / \mathbb{Z}$ for geometric representations of blow-ups $G$ of generalised Andrásfai graphs. Let $V$ be the set of points of the unit circle that are identified with the vertices of $G$. For an interval $I \subseteq \mathbb{R} / \mathbb{Z}$, we write $\lambda(I)$ for the number of vertices of $G$ contained in $I$, i.e.,

$$
\lambda(I)=|V \cap I| .
$$

This defines expressions such as $\lambda([a, b]), \lambda([a, b))$, etc.

Since subsets of $\lfloor n / 2\rfloor$ vertices are of special interest, we denote for every $\xi \in \mathbb{R} / \mathbb{Z}$ by $z_{\xi}$ the vertex from $V$ with the property

$$
\lambda\left(\left[\xi, z_{\xi}\right]\right)=\lfloor n / 2\rfloor .
$$

In the proof of Theorem 1.4 we shall use the following lemma and, since the proof will be carried out by contradiction, the graphs $G$ that we shall consider also satisfy the density assumption for parts $(i v)$ and $(v)$.

Lemma 2.2. For integers $k \geqslant 2$ and $d \geqslant 1$ let $G=(V, E)$ be a blow-up of the generalised Andrásfai graph $F_{d}^{k}$ having a geometric representation with $V \subseteq \mathbb{R} / \mathbb{Z}$ satisfying (2.1) and $|V|=n$. Then the following holds for every interval $I \subseteq \mathbb{R} / \mathbb{Z}$ :

( $i)$ If $I$ has length at most $\frac{k-1}{2 k-1}$, then $V \cap I$ is an independent set in $G$ and $\lambda(I) \leqslant \alpha(G)$.

(ii) If I has length at most $\frac{1}{2 k-1}$, then $\lambda(I) \leqslant(2 k-3) \alpha(G)-(k-2) n$.

(iii) If I has length at least $\frac{1}{2 k-1}$, then $\lambda(I) \geqslant n-2 \alpha(G)$.

If in addition $G$ is $\left(\frac{1}{2}, \frac{1}{2(2 k+1)^{2}}\right)$-dense and $2(2 k+1) \mid n$, then the following holds for $\xi \in \mathbb{R} / \mathbb{Z}$ : 
(iv) If $\lambda\left(\left[\xi, \xi+\frac{k-1}{2 k-1}\right]\right)=\alpha(G)$, then $\lambda\left(\left[\xi, z_{\xi}-\frac{k-1}{2 k-1}\right)\right)>2 \alpha(G)-\frac{2 k-1}{2 k+1} n$.

(v) We have $\lambda\left(\left(\xi-\frac{1}{2 k-1}, \xi+\frac{1}{2 k-1}\right)\right)>\frac{4}{2 k+1} n-2 \lambda\left(\left(\xi+\frac{k-1}{2 k-1}, \xi-\frac{k-1}{2 k-1}\right)\right)$.

Proof. Part $(i)$ follows directly from the definition of the geometric representation in (2.1). For part $(i i)$ we note that

$$
(2 k-3) \frac{k-1}{2 k-1}=(k-2)+\frac{1}{2 k-1} .
$$

Consequently, there exist $2 k-3$ consecutive intervals of length $\frac{k-1}{2 k-1}$ that wrap $k-2$ times around $\mathbb{R} / \mathbb{Z}$ in such a way that only $I$ is covered $k-1$ times. Therefore, $(i)$ yields

$$
(2 k-3) \alpha(G) \geqslant(k-2) n+\lambda(I)
$$

and the desired estimate follows.

Part (iii) is also a consequence of $(i)$ and the observation that there are two intervals of length at most $\frac{k-1}{2 k-1}$ that together with $I$ cover $\mathbb{R} / \mathbb{Z}$ once.

In the proofs of parts $(i v)$ and $(v)$ we make use of the inequality

$$
\lambda\left(\left[\xi, z_{\xi}-\frac{k-1}{2 k-1}\right)\right)>\frac{2 n}{2 k+1}-2 \lambda\left(\left(\xi+\frac{k-1}{2 k-1}, z_{\xi}\right]\right),
$$

which we show first. For that we note that (2.1) implies

$$
e_{G}\left(\left[\xi, z_{\xi}\right] \cap V\right) \leqslant \lambda\left(\left[\xi, z_{\xi}-\frac{k-1}{2 k-1}\right)\right) \cdot \lambda\left(\left(\xi+\frac{k-1}{2 k-1}, z_{\xi}\right]\right) .
$$

Hence, the additional assumption that $G$ is $\left(\frac{1}{2}, \frac{1}{2(2 k+1)^{2}}\right)$-dense combined with the simplest case of the inequality between the arithmetic and geometric mean yields

$$
\left(\frac{n}{2 k+1}\right)^{2}<2 e_{G}\left(\left[\xi, z_{\xi}\right] \cap V\right) \leqslant \frac{1}{4}\left(\lambda\left(\left[\xi, z_{\xi}-\frac{k-1}{2 k-1}\right)\right)+2 \lambda\left(\left(\xi+\frac{k-1}{2 k-1}, z_{\xi}\right]\right)\right)^{2}
$$

which establishes (2.4).

The remaining parts $(i v)$ and $(v)$ follow from (2.4). In fact, for $(i v)$ the additional assumption $\lambda\left(\left[\xi, \xi+\frac{k-1}{2 k-1}\right]\right)=\alpha(G)$ yields $\lambda\left(\left(\xi+\frac{k-1}{2 k-1}, z_{\xi}\right]\right)=n / 2-\alpha(G)$ and, hence, $(i v)$ follows from $(2.4)$.

For the proof of $(v)$ we will apply (2.4) twice. First we apply it for the given $\xi \in \mathbb{R} / \mathbb{Z}$ and, since by $(i)$ we also have $z_{\xi} \in\left(\xi+\frac{k-1}{2 k-1}, \xi+\frac{k}{2 k-1}\right)$, we obtain

$$
\lambda\left(\left[\xi, \xi+\frac{1}{2 k-1}\right)\right) \geqslant \lambda\left(\left[\xi, z_{\xi}-\frac{k-1}{2 k-1}\right)\right) \stackrel{(2.4)}{>} \frac{2 n}{2 k+1}-2 \lambda\left(\left(\xi+\frac{k-1}{2 k-1}, z_{\xi}\right]\right) .
$$

The second symmetric application of $(2.4)$ in $-\mathbb{R} / \mathbb{Z}$ to $-\xi$ yields

$$
\lambda\left(\left(\xi-\frac{1}{2 k-1}, \xi\right]\right) \stackrel{(2.4)}{>} \frac{2 n}{2 k+1}-2 \lambda\left(\left[z_{\xi}^{\prime}, \xi-\frac{k-1}{2 k-1}\right)\right),
$$

for $z_{\xi}^{\prime} \in\left(z_{\xi}, \xi\right)$ with $\lambda\left(\left[z_{\xi}^{\prime}, \xi\right]\right)=n / 2$. Consequently, if $\xi \notin V$ then summing the inequalities (2.5) and (2.6) yields part $(v)$. However, if $\xi \in V$ then still the same conclusion follows, 
since $(2 k+1) \mid n$ implies that the right-hand sides of (2.5) and (2.6) are integers and both inequalities are strict.

\section{§3. Blow-UPS OF GENERALised ANDRÁSFAi graphS}

In this section we establish Theorem 1.4. For that it suffices to show that blow-ups $G$ of generalised Andrásfai graphs $F_{d}^{k}$ are not $\left(\frac{1}{2}, \frac{1}{2(2 k+1)^{2}}\right)$-dense and we will appeal to the geometric representation from Lemma 2.1 of such graphs. The strategy of our proofs is that we try to find an interval of consecutive vertices spanning few edges. To this end we distinguish two cases depending on the independence number $\alpha(G)$ and start with the case that $\alpha(G)$ is not too large.

Proposition 3.1. If $G$ is a blow-up of a generalised Andrásfai graph $F_{d}^{k}$ for some integers $k \geqslant 2$ and $d \geqslant 1$ with $|V(G)|=n$ and $\alpha(G)<\frac{k}{2 k+1} n$, then $G$ is not $\left(\frac{1}{2}, \frac{1}{2(2 k+1)^{2}}\right)$-dense.

Proof. Let $G$ be a blow-up of $F_{d}^{k}$ with $|V(G)|=n$ and $\alpha(G)<\frac{k}{2 k+1} n$. Without loss of generality we may assume that $n$ is divisible by $2(2 k+1)$. This follows from the observation, that a graph $G$ is $\left(\frac{1}{2}, \frac{1}{2(2 k+1)^{2}}\right)$-dense if and only if the balanced blow-up of $G$ obtained by replacing each vertex by $2(2 k+1)$ vertices is $\left(\frac{1}{2}, \frac{1}{2(2 k+1)^{2}}\right)$-dense.

Suppose for the sake of contradiction that $G$ is $\left(\frac{1}{2}, \frac{1}{2(2 k+1)^{2}}\right)$-dense. From now on consider the geometric representation of $G$ given by Lemma 2.1. Let $V$ be the set of points of the unit circle that are identified with the vertices of $G$. Recall that in (2.2) we defined $\lambda(I)$ as the number of vertices contained in an interval $I \subseteq \mathbb{R} / \mathbb{Z}$. It will sometimes be convenient to count vertices on the boundary of an interval only with weight $1 / 2$. For that we write terms like $\lambda(\langle a, b\rangle), \lambda(\langle a, b))$, where the brackets " $\langle$ " or " $\rangle$ " mark that the left or right end-point of the respective interval is only counted $1 / 2$ if it is a vertex. Also recall that for $\xi \in \mathbb{R} / \mathbb{Z}$ we defined $z_{\xi} \in V$ in (2.3). Since by our assumption $\alpha(G)<n / 2$, we infer from part $(i)$ of Lemma 2.2 that

$$
z_{\xi} \in\left(\xi+\frac{k-1}{2 k-1}, \xi+\frac{k}{2 k-1}\right)
$$

which yields together with Lemma $2.2(i)$ that

$$
\sum_{x \in V \cap\left[\xi, z_{\xi}-\frac{k-1}{2 k-1}\right)}\left|N_{G}(x) \cap\left(x, z_{\xi}\right]\right|=e_{G}\left(\left[\xi, z_{\xi}\right] \cap V\right) .
$$

Moreover, part $(i i)$ of Lemma 2.2 applied to intervals $\left[x+\frac{k-1}{2 k-1}, x+\frac{k}{2 k-1}\right]$ combined with the assumption $\alpha(G)<\frac{k}{2 k+1} n$ leads to

$$
\begin{aligned}
\lambda\left(\left\langlex+\frac{k-1}{2 k-1}, x+\right.\right. & \left.\left.\frac{k}{2 k-1}\right\rangle\right) \leqslant \lambda\left(\left[x+\frac{k-1}{2 k-1}, x+\frac{k}{2 k-1}\right]\right) \\
& \leqslant(2 k-3) \alpha(G)-(k-2) n<(2 k-3) \frac{k}{2 k+1} n-(k-2) n=\frac{2}{2 k+1} n
\end{aligned}
$$


for every vertex $x \in V$. Consequently,

$$
\begin{aligned}
\sum_{x \in V}\left(\lambda\left(\left\langle x-\frac{k-1}{2 k-1}, x\right\rangle\right)+\lambda\left(\left\langle x, x+\frac{k-1}{2 k-1}\right\rangle\right)\right) & =\sum_{x \in V}\left(\lambda(\langle x, x+1\rangle)-\lambda\left(\left\langle x+\frac{k-1}{2 k-1}, x+\frac{k}{2 k-1}\right\rangle\right)\right) \\
& >n^{2}-\frac{2}{2 k+1} n^{2}=\frac{2 k-1}{2 k+1} n^{2}
\end{aligned}
$$

and by symmetry we may assume that

$$
\sum_{x \in V} \lambda\left(\left\langle x, x+\frac{k-1}{2 k-1}\right\rangle\right)>\frac{1}{2} \cdot \frac{2 k-1}{2 k+1} n^{2} .
$$

In view of (3.3) the following claim seems a bit surprising and, in fact, it will lead to the desired contradiction. For a simpler notation we set

$$
V_{\xi}=V \cap\left[\xi, z_{\xi}-\frac{k-1}{2 k-1}\right)
$$

for $\xi \in \mathbb{R} / \mathbb{Z}$.

Claim 3.2. For every $\xi \in \mathbb{R} / \mathbb{Z}$ we have

$$
\sum_{x \in V_{\xi}}\left(\lambda\left(\left\langle x, x+\frac{k-1}{2 k-1}\right\rangle\right)-\frac{1}{2} \cdot \frac{2 k-1}{2 k+1} n\right)<0 .
$$

Proof of Claim 3.2. Fix some $\xi \in \mathbb{R} / \mathbb{Z}$. Since we assume that $G$ is $\left(\frac{1}{2}, \frac{1}{2(2 k+1)^{2}}\right)$-dense, we have

$$
\sum_{x \in V_{\xi}} \lambda\left(\left(x+\frac{k-1}{2 k-1}, z_{\xi}\right]\right)=\sum_{x \in V_{\xi}}\left|N_{G}(x) \cap\left(x, z_{\xi}\right]\right| \stackrel{(3.2)}{=} e_{G}\left(\left[\xi, z_{\xi}\right] \cap V\right)>\frac{n^{2}}{2(2 k+1)^{2}} .
$$

Therefore,

$$
\begin{aligned}
\sum_{x \in V_{\xi}} \lambda\left(\left\langle x, x+\frac{k-1}{2 k-1}\right]\right) & =\sum_{x \in V_{\xi}}\left(\lambda\left(\left\langle x, z_{\xi}\right]\right)-\lambda\left(\left(x+\frac{k-1}{2 k-1}, z_{\xi}\right]\right)\right) \\
& <\sum_{x \in V_{\xi}} \lambda\left(\left\langle x, z_{\xi}\right]\right)-\frac{n^{2}}{2(2 k+1)^{2}} \\
& =\sum_{x \in V_{\xi}}\left(\lambda\left(\left[\xi, z_{\xi}\right]\right)-\lambda([\xi, x\rangle)\right)-\frac{n^{2}}{2(2 k+1)^{2}} \\
& \stackrel{(2.3)}{=}\left|V_{\xi}\right| \cdot \frac{n}{2}-\frac{n^{2}}{2(2 k+1)^{2}}-\sum_{x \in V_{\xi}} \lambda([\xi, x\rangle) .
\end{aligned}
$$

We observe

$$
\sum_{x \in V_{\xi}} \lambda([\xi, x\rangle)=\sum_{i=1}^{\left|V_{\xi}\right|}\left(i-\frac{1}{2}\right)=\frac{\left|V_{\xi}\right|^{2}}{2}
$$


and combining (3.5) and (3.6) yields

$$
\begin{aligned}
\sum_{x \in V_{\xi}}\left(\lambda\left(\left\langle x, x+\frac{k-1}{2 k-1}\right]\right)-\frac{1}{2} \cdot \frac{2 k-1}{2 k+1} n\right) & <\left|V_{\xi}\right| \cdot\left(\frac{n}{2}-\frac{1}{2} \cdot \frac{2 k-1}{2 k+1} n\right)-\frac{n^{2}}{2(2 k+1)^{2}}-\frac{\left|V_{\xi}\right|^{2}}{2} \\
& =-\frac{1}{2}\left(\left|V_{\xi}\right|-\frac{n}{2 k+1}\right)^{2} \leqslant 0
\end{aligned}
$$

which establishes the claim.

Now set $V^{*}=\left\{\xi \in \mathbb{R} / \mathbb{Z}: \xi+\frac{k-1}{2 k-1} \in V\right\}$. Starting with an arbitrary $x(0) \in V^{*}$ we define recursively a sequence of members of $V^{*}$ by putting

$$
x(i+1)=z_{x(i)}-\frac{k-1}{2 k-1}
$$

for every $i \in \mathbb{N}$. Since $V^{*}$ is finite, this sequence is eventually periodic and thus we could have chosen $x(0)$ such that $x(m)=x(0)$ holds for some $m \geqslant 2$. Let $w \in \mathbb{N}$ denote the number of times we wind around the circle when reaching $x(m)$ from $x(0)$ by this construction. By Claim 3.2 we know that

$$
\sum_{i=0}^{m-1} \sum_{x \in V_{x(i)}}\left(\lambda\left(\left\langle x, x+\frac{k-1}{2 k-1}\right\rangle\right)-\frac{1}{2} \cdot \frac{2 k-1}{2 k+1} n\right)<0 .
$$

On the other hand, (3.3) yields

$$
\begin{aligned}
& \sum_{i=0}^{m-1} \sum_{x \in V_{x(i)}}\left(\lambda\left(\left\langle x, x+\frac{k-1}{2 k-1}\right\rangle\right)-\frac{1}{2} \cdot \frac{2 k-1}{2 k+1} n\right) \stackrel{(3.4)}{=} w \cdot \sum_{x \in V}\left(\lambda\left(\left\langle x, x+\frac{k-1}{2 k-1}\right\rangle\right)-\frac{1}{2} \cdot \frac{2 k-1}{2 k+1} n\right) \\
& \stackrel{(3.3)}{>} w \cdot\left(\frac{1}{2} \cdot \frac{2 k-1}{2 k+1} n^{2}-\frac{1}{2} \cdot \frac{2 k-1}{2 k+1} n^{2}\right)=0,
\end{aligned}
$$

which is a contradiction and concludes the proof of Proposition 3.1.

It is left to consider the case when $G$ contains a large independent set.

Proposition 3.3. If $G$ is a blow-up of a generalised Andrásfai graph $F_{d}^{k}$ for some integers $k \geqslant 2$ and $d \geqslant 1$ with $|V(G)|=n$ and $\alpha(G) \geqslant \frac{k}{2 k+1} n$, then $G$ is not $\left(\frac{1}{2}, \frac{1}{2(2 k+1)^{2}}\right)$-dense.

Proof. Similarly as in the proof of Proposition 3.1 we consider the geometric representation of an $n$-vertex graph $G$ that is a blow-up of a generalised Andrásfai graph $F_{d}^{k}$ and identify the vertex set of $G$ with some set $V \subseteq \mathbb{R} / \mathbb{Z}$ so that (2.1) holds. Again we may assume without loss of generality that $n$ is divisible by $2(2 k+1)$ and we suppose for a contradiction that $G$ is $\left(\frac{1}{2}, \frac{1}{2(2 k+1)^{2}}\right)$-dense. In particular, $\alpha(G)<n / 2$ and the additional assumptions for parts $(i v)$ and $(v)$ of Lemma 2.2 are satisfied. 
Observe that every independent set of $G$ is contained in some interval of $\mathbb{R} / \mathbb{Z}$ of length $\frac{k-1}{2 k-1}$. Therefore, without loss of generality we may assume that $\left[0, \frac{k-1}{2 k-1}\right]$ contains a maximum independent set, i.e.,

$$
\lambda\left(\left[0, \frac{k-1}{2 k-1}\right]\right)=\alpha(G) \geqslant \frac{k}{2 k+1} n .
$$

Recall that in $(2.3)$ we defined a point $z_{0}$ with $\lambda\left(\left[0, z_{0}\right]\right)=n / 2$. Let the vertex $z^{\prime}$ be defined similarly by $\lambda\left(\left[z^{\prime}, \frac{k-1}{2 k-1}\right]\right)=n / 2$. Then we have

$$
\begin{aligned}
\lambda\left(\left(z_{0}, z^{\prime}\right)\right) & =n-\lambda\left(\left[z^{\prime}, 0\right)\right)-\lambda\left(\left[0, \frac{k-1}{2 k-1}\right]\right)-\lambda\left(\left(\frac{k-1}{2 k-1}, z_{0}\right]\right) \\
& =n-(n / 2-\alpha(G))-\alpha(G)-(n / 2-\alpha(G)) \\
& =\alpha(G)
\end{aligned}
$$

and since $z_{0}, z^{\prime} \in V$ the maximality of $\alpha(G)$ discloses that the interval $\left[z_{0}, z^{\prime}\right]$ has at least the length $\frac{k-1}{2 k-1}$. Hence there is a closed subinterval $\left[b_{k}, b_{0}\right]$ of $\left[z_{0}, z^{\prime}\right]$ whose length is exactly $\frac{k-1}{2 k-1}$. We complete $b_{0}$ and $b_{k}$ to the vertices of a regular $(2 k-1)$-gon, i.e., we consider the points $b_{i} \in \mathbb{R} / \mathbb{Z}$ for $i \in\{0, \ldots, 2 k-2\}$ such that the intervals $\left[b_{i}, b_{i+1}\right]$ have length $\frac{1}{2 k-1}$ (see Figure 4 ). Notice that $\alpha(G)<n / 2$ entails

$$
z_{0} \in\left(b_{k-1}, b_{k}\right] .
$$

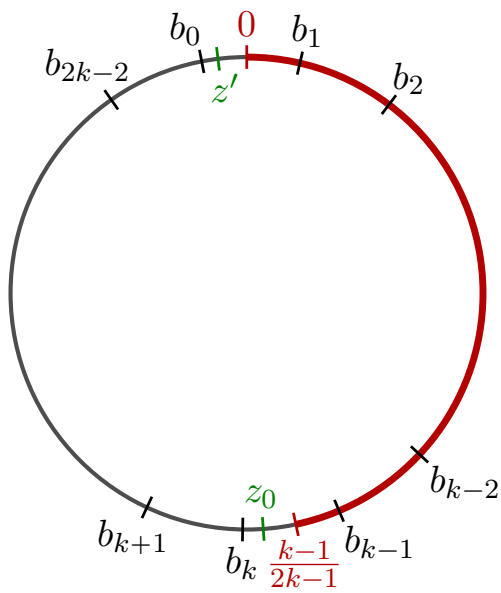

Figure 4. Largest independent set of $G$ is contained in the interval $\left[0, \frac{k-1}{2 k-1}\right]$ and the intervals $\left[0, z_{0}\right],\left[z^{\prime}, \frac{k-1}{2 k-1}\right]$ contain $n / 2$ vertices each. The $b_{i}$ form a regular $(2 k-1)$-gon.

Below we apply Lemma 2.2 to obtain several bounds on $\lambda\left(\left[b_{1}, b_{k-1}\right]\right)$ and $\lambda\left(\left[b_{k+1}, b_{2 k-2}\right]\right)$ that eventually lead to the desired contradiction. Applying Lemma $2.2(i v)$ with $\xi=0$ gives

$$
\lambda\left(\left[0, b_{1}\right)\right)=\lambda\left(\left[0, b_{k}-\frac{k-1}{2 k-1}\right)\right) \stackrel{(3.7)}{\geqslant} \lambda\left(\left[0, z_{0}-\frac{k-1}{2 k-1}\right)\right)>2 \alpha(G)-\frac{2 k-1}{2 k+1} n
$$


and, by symmetry, we also have

$$
\lambda\left(\left(b_{k-1}, \frac{k-1}{2 k-1}\right]\right)>2 \alpha(G)-\frac{2 k-1}{2 k+1} n .
$$

Consequently, we arrive at

$$
\begin{aligned}
\lambda\left(\left[b_{1}, b_{k-1}\right]\right) & =\lambda\left(\left[0, \frac{k-1}{2 k-1}\right]\right)-\lambda\left(\left[0, b_{1}\right)\right)-\lambda\left(\left(b_{k-1}, \frac{k-1}{2 k-1}\right]\right) \\
& <\alpha(G)-2\left(2 \alpha(G)-\frac{2 k-1}{2 k+1} n\right)=\frac{4 k-2}{2 k+1} n-3 \alpha(G) .
\end{aligned}
$$

In particular, for the case $k=2$ this implies

$$
0 \leqslant \lambda\left(\left[b_{1}, b_{1}\right]\right)<\frac{6}{5} n-3 \alpha(G),
$$

which contradicts our assumption $\alpha(G) \geqslant 2 n / 5$. Similarly, for $k=3$ inequality (3.8) combined with Lemma 2.2 (iii) gives

$$
n-2 \alpha(G) \leqslant \lambda\left(\left[b_{1}, b_{2}\right]\right)<\frac{10}{7} n-3 \alpha(G),
$$

which again contradicts the assumption $\alpha(G) \geqslant 3 n / 7$ of this case. Consequently, for the rest of the proof we can assume that $k \geqslant 4$.

Next we note that both intervals $\left(b_{k-1}, b_{2 k-2}\right)$ and $\left(b_{k+1}, b_{1}\right)$ have length $\frac{k-1}{2 k-1}$ and, hence, Lemma $2.2(i)$ implies

$$
\lambda\left(\left(b_{k-1}, b_{2 k-2}\right)\right)+\lambda\left(\left(b_{k+1}, b_{1}\right)\right) \leqslant 2 \alpha(G)
$$

and, therefore,

$$
\lambda\left(\left(b_{k+1}, b_{2 k-2}\right)\right) \leqslant 2 \alpha(G)-\lambda\left(\left(b_{k-1}, b_{1}\right)\right)=2 \alpha(G)-\left(n-\lambda\left(\left[b_{1}, b_{k-1}\right]\right)\right) .
$$

Finally, below we will verify

$$
\frac{4 k-5}{2 k+1} n-2 \alpha(G)-\lambda\left(\left[b_{1}, b_{k-1}\right]\right)<\lambda\left(\left(b_{k+1}, b_{2 k-2}\right)\right) .
$$

Before we prove (3.10), we note that using (3.9) as an upper bound for the right-hand side of (3.10) leads to

$$
\frac{6 k-4}{2 k+1} n-4 \alpha(G)<2 \lambda\left(\left[b_{1}, b_{k-1}\right]\right) \stackrel{(3.8)}{<} \frac{8 k-4}{2 k+1} n-6 \alpha(G) .
$$

This inequality contradicts the assumption $\alpha(G) \geqslant \frac{k}{2 k+1}$ of the proposition and, hence, we conclude the proof by establishing (3.10).

For the proof of inequality (3.10) we appeal to Lemma $2.2(v)$ with $\xi=b_{i}$ for every $i=2, \ldots, k-2$. We set

$$
I_{i}=\left(b_{i}-\frac{1}{2 k-1}, b_{i}+\frac{1}{2 k-1}\right)=\left(b_{i-1}, b_{i+1}\right)
$$


and then in view of

$$
\left(b_{i}+\frac{k-1}{2 k-1}, b_{i}-\frac{k-1}{2 k-1}\right)=\left(b_{i+k-1}, b_{i+k}\right)
$$

part $(v)$ translates to

$$
\lambda\left(I_{i}\right)>\frac{4}{2 k+1} n-2 \lambda\left(\left(b_{i+k-1}, b_{i+k}\right)\right) .
$$

Furthermore, we note that for every $i \in\{2, \ldots, k-2\}$ we have $I_{i} \subseteq\left[b_{1}, b_{k-1}\right]$ and each of the two families

$$
\mathcal{I}_{0}=\left\{I_{i}: i \text { even and } 2 \leqslant i \leqslant k-2\right\} \quad \text { and } \quad \mathcal{I}_{1}=\left\{I_{i}: i \text { odd and } 2 \leqslant i \leqslant k-2\right\}
$$

consists of mutually disjoint intervals. Moreover, we can add the interval $\left[b_{1}, b_{2}\right)$ to $\mathcal{I}_{1}$ and $\left(b_{k-2}, b_{k-1}\right]$ either to $\mathcal{I}_{1}$ (when $k$ is even) or to $\mathcal{I}_{0}$ (when $k$ is odd) and still each family consists of mutually disjoint intervals all contained in $\left[b_{1}, b_{k-1}\right]$. As a result we get

$$
2 \lambda\left(\left[b_{1}, b_{k-1}\right]\right) \geqslant \lambda\left(\left[b_{1}, b_{2}\right)\right)+\sum_{i=2}^{k-2} \lambda\left(I_{i}\right)+\lambda\left(\left(b_{k-2}, b_{k-1}\right]\right) .
$$

Moreover, using the estimate from Lemma $2.2($ iii $)$ for $\lambda\left(\left[b_{1}, b_{2}\right)\right)$ and $\lambda\left(\left(b_{k-2}, b_{k-1}\right]\right)$ and (3.11) for every term in the middle sum, we arrive at

$$
\begin{aligned}
2 \lambda\left(\left[b_{1}, b_{k-1}\right]\right)> & (n-2 \alpha(G))+\sum_{i=2}^{k-2}\left(\frac{4 n}{2 k+1}-2 \lambda\left(\left(b_{i+k-1}, b_{i+k}\right)\right)\right)+(n-2 \alpha(G)) \\
\geqslant & 2 n-4 \alpha(G)+(k-3) \cdot \frac{4 n}{2 k+1}-2 \lambda\left(\left(b_{k+1}, b_{2 k-2}\right)\right) \\
& =\frac{8 k-10}{2 k+1} n-4 \alpha(G)-2 \lambda\left(\left(b_{k+1}, b_{2 k-2}\right)\right) .
\end{aligned}
$$

Rearranging the last inequality gives (3.10) and this concludes the proof.

\section{REFERENCES}

[1] B. Andrásfai, Über ein Extremalproblem der Graphentheorie, Acta Math. Acad. Sci. Hungar. 13 (1962), 443-455 (German). MR0145503 ^1.1, 1.1, 2

[2] _ Graphentheoretische Extremalprobleme, Acta Math. Acad. Sci. Hungar 15 (1964), 413-438 (German). MR0169227 $\uparrow 1.1,1.1$

[3] B. Andrásfai, P. Erdős, and V. T. Sós, On the connection between chromatic number, maximal clique and minimal degree of a graph, Discrete Math. 8 (1974), 205-218. MR0340075 $\uparrow 1.1$

[4] C. C. Chen, G. P. Jin, and K. M. Koh, Triangle-free graphs with large degree, Combin. Probab. Comput. 6 (1997), no. 4, 381-396, DOI 10.1017/S0963548397003167. MR1483425 ^1.1, 1.1

[5] P. Erdős, Remarks on a theorem of Ramsay, Bull. Res. Council Israel. Sect. F 7F (1957/1958), 21-24. MR0104594 $\uparrow 1.1,1.1,2$

[6] P. Erdős, Problems and results in graph theory and combinatorial analysis, Proceedings of the Fifth British Combinatorial Conference (Univ. Aberdeen, Aberdeen, 1975), Utilitas Math., Winnipeg, Man., 1976, pp. 169-192. Congressus Numerantium, No. XV. MR0409246 ^1 
[7] P. Erdős, Some old and new problems in various branches of combinatorics, Discrete Math. 165/166 (1997), 227-231, DOI 10.1016/S0012-365X(96)00173-2. Graphs and combinatorics (Marseille, 1995). MR1439273 11,1

[8] P. Erdős, R. J. Faudree, C. C. Rousseau, and R. H. Schelp, A local density condition for triangles, Discrete Math. 127 (1994), no. 1-3, 153-161, DOI 10.1016/0012-365X(92)00474-6. Graph theory and applications (Hakone, 1990). MR1273598 11

[9] R. Häggkvist, Odd cycles of specified length in nonbipartite graphs, Graph theory (Cambridge, 1981), North-Holland Math. Stud., vol. 62, North-Holland, Amsterdam-New York, 1982, pp. 89-99. MR671908 $\uparrow 1.1$

[10] G. P. Jin, Triangle-free four-chromatic graphs, Discrete Math. 145 (1995), no. 1-3, 151-170, DOI 10.1016/0012-365X(94)00063-O. MR1356592 ^1.1, 1.1

[11] P. Keevash and B. Sudakov, Sparse halves in triangle-free graphs, J. Combin. Theory Ser. B 96 (2006), no. 4, 614-620, DOI 10.1016/j.jctb.2005.11.003. MR2232396 $\uparrow 1.1$

[12] M. Krivelevich, On the edge distribution in triangle-free graphs, J. Combin. Theory Ser. B 63 (1995), no. 2, 245-260, DOI 10.1006/jctb.1995.1018. MR1320169 ^1, 1, 1.1

[13] S. Letzter and R. Snyder, The homomorphism threshold of $\left\{C_{3}, C_{5}\right\}$-free graphs, available at arXiv:1610.04932. Submitted. $\uparrow 1.2$

[14] S. Norin and L. Yepremyan, Sparse halves in dense triangle-free graphs, J. Combin. Theory Ser. B 115 (2015), 1-25, DOI 10.1016/j.jctb.2015.04.006. MR3383248 ^1.1, 1.1

[15] D. R. Woodall, The binding number of a graph and its Anderson number, J. Combinatorial Theory Ser. B 15 (1973), 225-255. MR0327573 $\uparrow 1.1$

Fachbereich Mathematik, Universität Hamburg, Hamburg, Germany

E-mail address: Wiebke.Bedenknecht@uni-hamburg.de

E-mail address: Christian.Reiher@uni-hamburg.de

E-mail address: schacht@math.uni-hamburg.de

Instituto de Matemática e Estatística, Universidade de São Paulo, São Paulo, Brazil E-mail address: mota@ime.usp.br 